\title{
Cardiomyocyte-Driven Actuation in Biohybrid Microcylinders
}

\author{
Jaewon Yoon, Tom W. Eyster, Asish C. Misra, and Joerg Lahann*
}

Among many microactuator designs that have been developed and studied in recent years, bioactuators or biohybrid actuators are especially interesting due to the exploitation of living cells, their self-actuating behavior, and the potential promise for in vivo applications. ${ }^{[1-3]}$ For example, integrating the spontaneous contraction of cardiomyocytes into complex polymer structures could generate spontaneous motion, only requiring a natural energy source such as glucose along with standard cell media. ${ }^{[4]}$ To improve the performance of the actuating behavior of cells, recent work has involved the incorporation of carbon-based materials such as carbon nanotubes and graphene. ${ }^{[5,6]}$ Because of their unique electrical conductivity and high mechanical properties, many reports have exploited the use of these materials to modulate electroactive tissue constructs or as a reinforcing agent for soft hydrogels. ${ }^{[7,8]}$ Despite the importance of this work, biocompatibility and toxicity of carbon-based materials still remain unknown. Additionally, the fabrication of artificial bioactuators becomes difficult at smaller scales and when 3D structures are involved. With recent advances in the field of polymer engineering, there has been improved control of the $3 \mathrm{D}$ cell culture environment. ${ }^{[9-12]}$ Various nano- and microfabrication techniques provide scaffolds with diverse scope of size, shape, or topology. ${ }^{[11,13-15]} 3 \mathrm{D}$ structures are studied instead of merely 2D films and the effect of pore architectures on regulating cells is also under intense scrutiny. ${ }^{[16-18]}$ Moreover, current research on the construction of microstructures with tunable functionalities has enabled direct control of cell behaviors such as cell adhesion, migration and proliferation. ${ }^{[19,20]}$ In fact, a broad range of investigations have reported on engineering artificial cell culture matrices with specific functions such as being bioactive, ${ }^{[15,21]}$ nonfouling, ${ }^{[22-24]}$ or possessing stimuli-responsive ${ }^{[20,25]}$ properties.

When creating bioactuators powered by cell movement, cell alignment on the device becomes a critical design component for maximizing the output force. ${ }^{[7]}$ As a strategy for

Dr. J. Yoon, Prof. J. Lahann

Macromolecular Science and Engineering Program

University of Michigan

Ann Arbor, MI 48109, USA

E-mail: lahann@umich.edu

Dr. T. W. Eyster, Prof. J. Lahann

Department of Chemical Engineering

University of Michigan

Ann Arbor, MI 48109, USA

Dr. A. C. Misra, Prof. J. Lahann

Department of Biomedical Engineering

University of Michigan

Ann Arbor, MI 48109, USA

Prof. J. Lahann

Biointerfaces Institute

University of Michigan

Ann Arbor, MI 48109, USA

DOI: 10.1002/adma.201501284 aligning cells, microfabrication techniques have been widely investigated to build surfaces with varying topologies. ${ }^{[26-28]}$ For example, cardiomyocytes have been seeded on both grooved and flat surfaces, and cells cultured on the grooved pattern were more effective in aligning themselves, whereas the flat surface leads to randomly adhered cells with a low level of intracellular organization. ${ }^{[27,28]}$ In addition to these studies, several reports have focused on engineering 3D micropillars, ${ }^{[29]}$ micropumps, ${ }^{[30]}$ and microcantilevers ${ }^{[31-33]}$ as models for cardiac cell studies. Moreover, Feinberg et al. produced polydimethylsiloxane (PDMS) thin films containing cardiomyocytes in an arranged pattern and induced cell movement through directionally applied electric fields. ${ }^{[34]}$ Using a similar concept, the same group further developed a microstructure that resembles the movement of jellyfish. ${ }^{[35]}$ Other attempts have been made to improve cardiac cell culture platforms by incorporating hydrogels into cell culture substrates; Cvetcovic et al. utilizes 3D printing to build hydrogel/C2C12 skeletal muscle "biobots." [36,37] While such examples to date present meaningful improvements on the performance and usability of biointegrated structures, there is still a need for new types of microactuating devices that can further basic studies on in vitro 3D environments.

Here, we design and build compact and miniaturized biohybrid microcylinders that actuate in response to the spontaneous contraction of neonatal rat cardiomyocytes. We chose cardiac cells because they do not require application of an electric field for stimulation. Anisotropic microcylinders were created via electrohydrodynamic (EHD) cojetting, a technique which provides easy functional materials processing. ${ }^{[38]}$ We have previously reported bicompartmental poly(D,L-lactide-co-glycolide) (PLGA) microfibers through EHD cojetting that are capable of guiding cell adhesion on individual fibers. ${ }^{[15]}$ Through surface modification by covalent linkage of a cell adhesive peptide, we were able to demonstrate phase-selective cell attachment on a single microfiber. ${ }^{[15]}$ To construct a new class of synthetic bioactuators, we aim to guide cardiomyocytes onto single microcylinders and to demosntrate cell-induced bending of the microcylinder.

A schematic diagram illustrating the preparation of the microcylinders is shown in Figure 1. First, bicompartmental microfibers with a $20 \mu \mathrm{m}$ diameter were produced via EHD cojetting. While PLGA was chosen as a base material for both compartments, cyclooctyne-functionalized poly(lactic acid) (COT-PLA) was selectively introduced in one of the compartments. Cyclooctyne moieties are among the reactive groups commonly used in "click" chemistry under copper-free reaction conditions. ${ }^{[39]}$ To design microcylinders that can be actuated by controlled cell action, it was important to control the alignment of the cells along the long axis of the microcylinders. To this end, the incorporation of cyclooctyne groups in the microfibers was necessary for rendering specific regions of the microcylinders protein repellent. In addition to COT-PLA, 

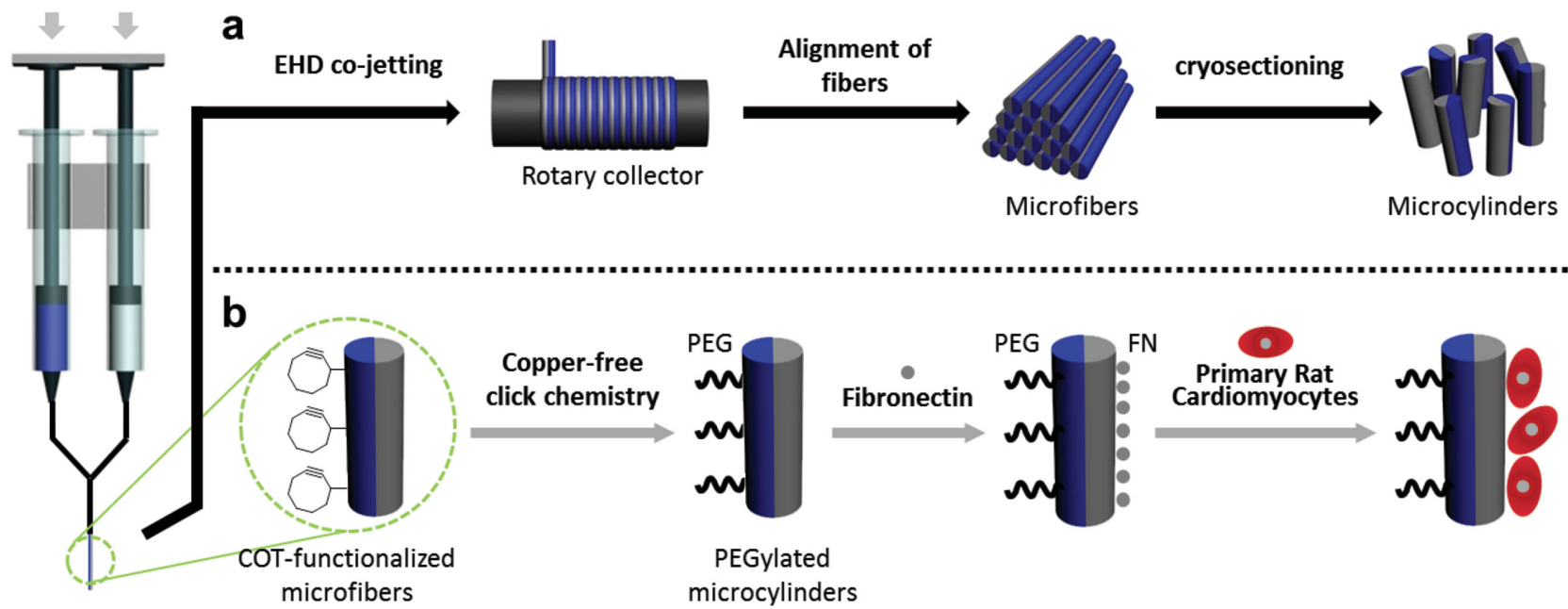

Figure 1. Fabrication of bicompartmental microcylinders for bioactuators. a) The first step involves collecting fibers as aligned bundles followed by cryosectioning at a desired length to prepare microcylinders. b) The second step is to utilize the obtained microcylinders for selective surface modification with nonfouling PEG molecules. The immobilization of PEG on the half surface of microcylinders results in selective protein adsorption as well as cell attachment.

the microcylinders also contained magnetite nanocrystals with an average diameter of $15 \mathrm{~nm}$. The addition of magnetite nanocrystals helped to separate the microcylinders from the cell culture, when replacing the cell media. Hence, the two solutions, PLGA/magnetite and COT-PLA/PLGA, were used to form well-defined bicompartmental microfibers through EHD cojetting and the resulting microfibers were further processed by automated microsectioning to yield uniform microcylinders following previously reported procedures. ${ }^{[38]}$ In order to confirm the selective localization of the cyclooctyne groups on the microcylinders, confocal Raman microspectroscopy was employed (Figure 2b). Raman analysis unambiguously confirmed that the presence of cyclooctyne groups was restricted to only one hemisphere. Once the spatially controlled loading of cyclooctyne groups was confirmed, we next immobilized nonfouling poly(ethylene glycol) (PEG) via click chemistry with azide-functionalized PEG.

As an initial step, longer microfibers were used in cell culture to ensure better attachment to the flat support substrates that allowed for easy handling during protein and cell assays. In order to impose directional protein adsorption and cell growth, azide-functionalized PEG molecules were selectively introduced to the COT-PLA-containing compartment. As an indirect method to prove successful binding of antifouling PEG on the surface, fluorescence-labeled bovine serum albumin (BSA, Alexa Fluor-647) was incubated with these PEG-modified microfibers for $3 \mathrm{~h}$ (Figure 2a). As PEG molecules are protein repellent, BSA adsorbed only to the compartment lacking the cyclooctyne groups (Figure 2a, center). This result was consistent with a reference experiment without COT-PLA polymers, which showed full coverage of BSA on the microfibers (Figure 2a, left). Likewise, shorter microcylinders modified with PEG were similarly selective in repelling BSA adsorption (Figure 2a, right).

To demonstrate the feasibility of these microfibers and microcylinders for selective cell attachment, NIH 3T3 fibroblasts were used. As seen in Figure 3, two different sets of microfibers, PLGA/PLGA and PLGA/PEG (after surface immobilization of PEG on COT-PLA), were utilized for the selective recruitment of cells. Due to the selective adsorption of celladhesive protein fibronectin (FN), the fibroblasts adhered and spread only on the compartment lacking PEG (Figure 3c). In contrast, PLGA/PLGA microfibers showed no selectivity on either the FN adsorption or the cell attachment (Figure 3b). Next, we incubated biocompartmental microcylinders with NIH 3T3 fibroblasts. Figure 3d demonstrates successfully aligned and adhered NIH 3T3 fibroblasts after $8 \mathrm{~h}$ of incubation.

Before attempting to pattern cardiomyocytes on bicompartmental microcylinders, the mechanical properties of the microcylinders had to be examined. PLGA is a biodegradable polymer which can be a good candidate for tissue engineering applications, ${ }^{[40]}$ but its high stiffness and low strain were a potential problem for the intended use in bioactuation. ${ }^{[41]}$ To overcome its limited mechanical properties, we prepared microcylinders composed of poly(lactide-co-caprolactone) (PLCL). In order to compare the mechanical properties of PLGA and PLCL microfibers, tensile tests of single fibers were performed. Based on the stress-strain curves presented in Figure S1 (Supporting Information), it was demonstrated that the PLGA microfibers had very low strain $(0.1 \pm 0.06)$ and higher Young's modulus $(1585 \pm 551 \mathrm{MPa})$ than PLGA/PLCL (strain: $7.4 \pm 1.3$, Young's modulus: $476 \pm 31 \mathrm{MPa}$ ) microfibers. PLCL shows a $T_{\mathrm{g}}$ of $\approx 16^{\circ} \mathrm{C}$ and it is in a rubbery state at room temperature, thus possessing a much higher strain value than PLGA. ${ }^{[42-44]}$ Moreover, when fracture strains for each microfiber $(n=10)$ were compared, PLGA/PLCL microfibers tend to have more than 70 times higher strain value than that of PLGA microfibers. With this in mind, PLGA/PLCL microcylinders were incubated with NIH3T3 fibroblasts to characterize cell adhesion. The selective adhesion of NIH3T3 fibroblasts was unambiguously demonstrated after surface modification of the microcylinders with PEG (Figure S2, Supporting Information). Furthermore, quantification analysis shows that the two data sets of the microcylinders before and after the posttreatment with PEG 
a
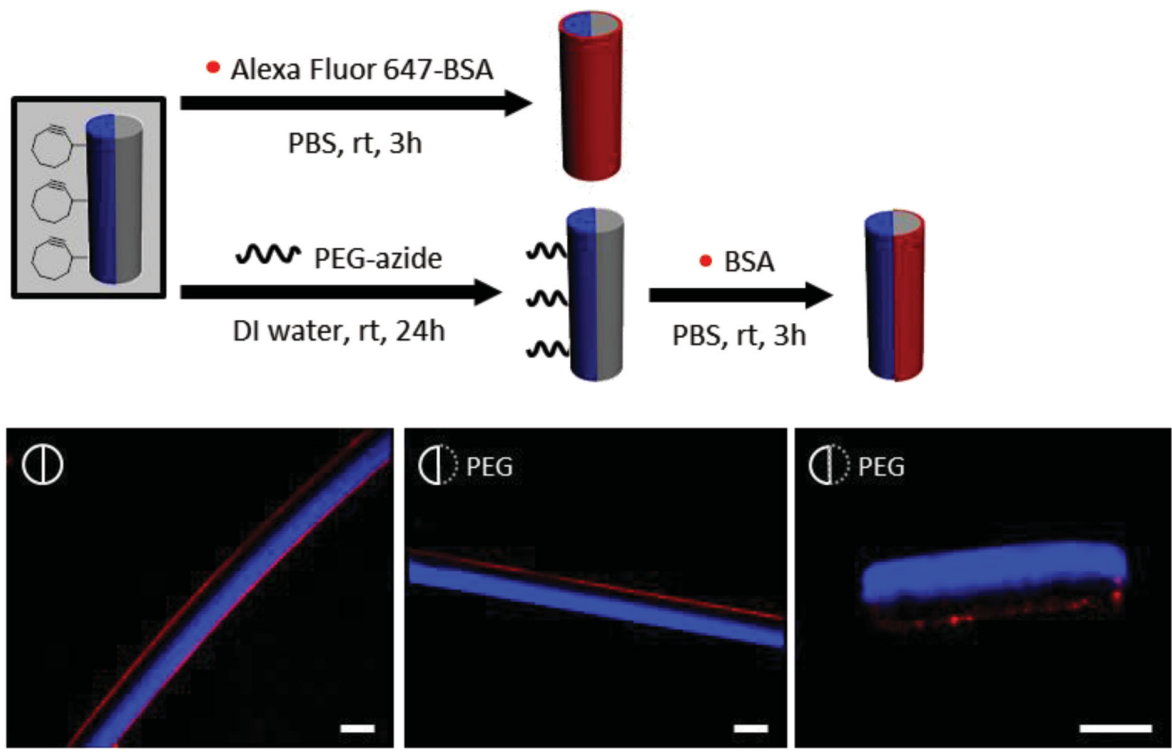

b
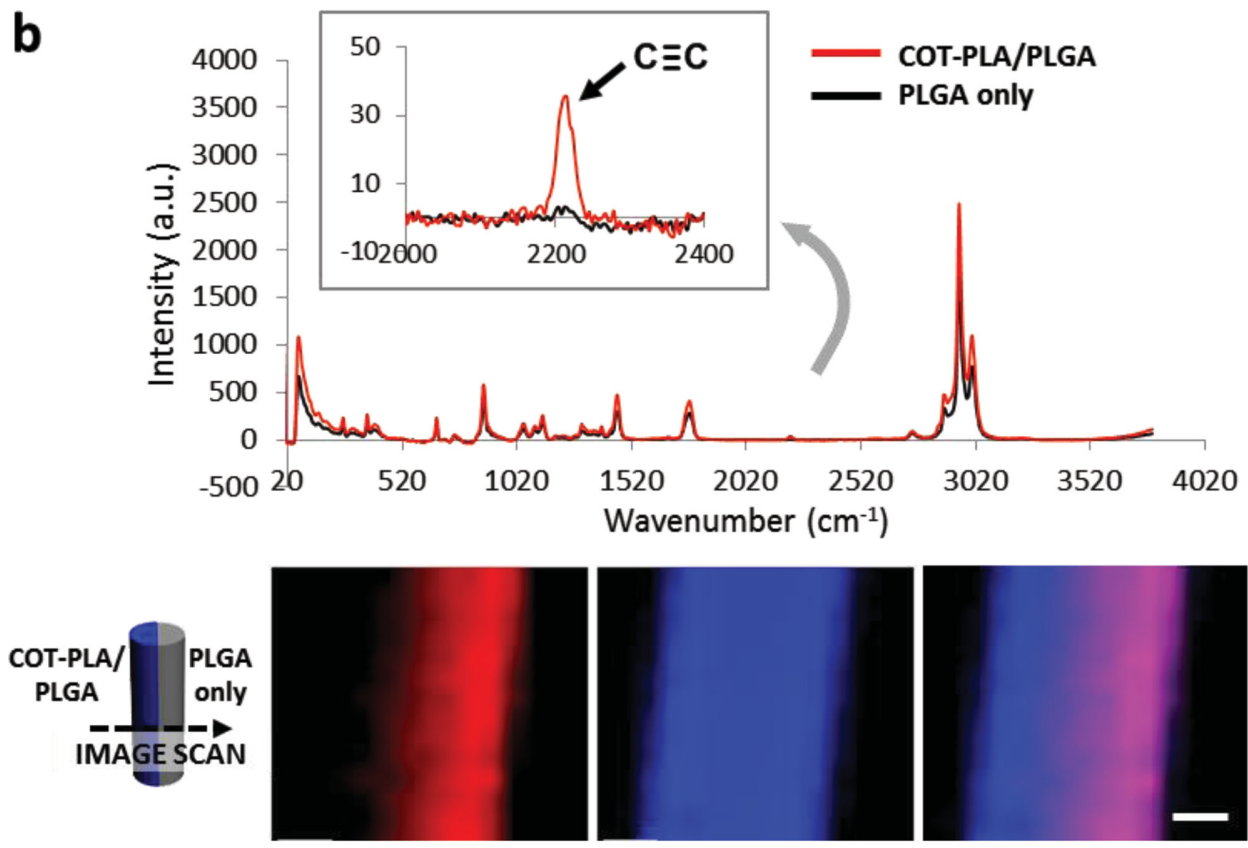

Figure 2. Characterization of COT-functionalized bicompartmental microcylinders. a) Selective adsorption of BSA on bicompartmental microfibers and microcylinders. CLSM images of the microfibers before the surface modification with PEG (left) shows BSA adsorbed to the entire fiber surface. After the selective surface modification with PEG (middle), BSA was adsorbed only on the unreacted surfaces. This result matched microcylinders (right), where the protein adsorption was prevented in the presence of the PEC chains. All scale bars are $20 \mu \mathrm{m}$. b) Confocal-Raman spectra obtained from the cross-sectional image scan of COT-functionalized bicompartmental microfibers. Characteristic peak near $2200 \mathrm{~cm}^{-1}$ shows triple bond of carbon, which indicates the presence of the COT groups in the half compartment. The overlaid image demonstrates the localization of the COT groups (red) in one compartment only. Scale bar is $5 \mu \mathrm{m}$.

gave a $p$-value $<0.05$, indicating that the PEGylated microcylinders significantly inhibit cell adhesion (Figure S2, Supporting Information).

Finally, to explore microcylinders as a biohybrid actuator, we studied neonatal cardiomyocytes, which spontaneously contract even in the absence of an external current. The neonatal cardiomyocytes were seeded on the PLGA/PLCL microcylinders. Figure 4 shows the selective cultivation of cardiomyocytes on PLGA/PLCL microcylinders treated with PEG. As shown before, FN selectively adsorbed on the hemisphere lacking PEG, which then promoted cell adhesion and spreading. The concept of having PEG selectively on the one compartment was very important for the realization of the bending behavior of the microcylinders, as if the cells are randomly arranged, it would be impossible to observe synchronized contraction and there would not be any significant net force. 
a
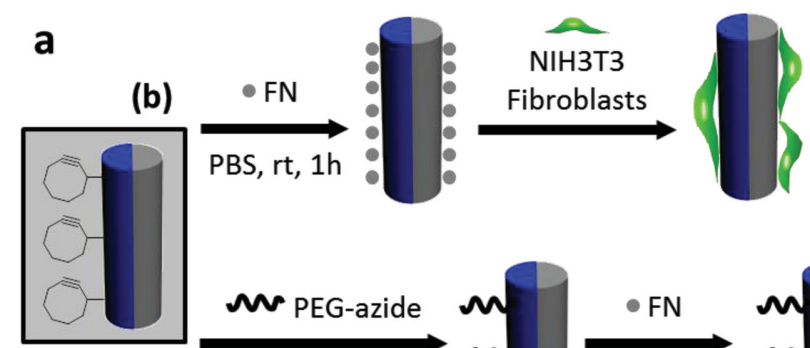

(c,d) DI-water, rt, 24h


Figure 3. Selective recruitment of NIH 3 T3 fibroblasts on bicompartmental microfibers and microcylinders. a) Two different sets of experimental schemes, with and without surface modification with PEG. b,c) CLSM images of bicompartmental microfibers correspond to each step. (b) indicates the microfibers before the surface modification with PEG (blue compartment), where the fibroblasts were introduced on both compartments. In contrast, when the microfibers were modified with PEG, the cell attachment was observed on non-PEGylated compartment only. d) CLSM images of bicompartmental microcylinders after surface modification with PEG (blue). The selective attachment of fibroblasts shows the presence of nonfouling PEG molecules in one half compartment. Scale bars in (b) and (c) are $50 \mu \mathrm{m}$ and (d) is $20 \mu \mathrm{m}$.

Upon establishing the selective binding of cardiomyocytes, we further investigated the bending behavior of the obtained microcylinders for potential actuating applications. The autonomous beating of the cells was observed after $2 \mathrm{~d}$ in rat cardiomyocytes growth media. The actuating behavior of the microcylinders was then investigated on the third day of the experiment. Movies recorded for $25 \mathrm{~s}$ revealed that microcylinders that were seeded with well-arranged cardiomyocytes

a
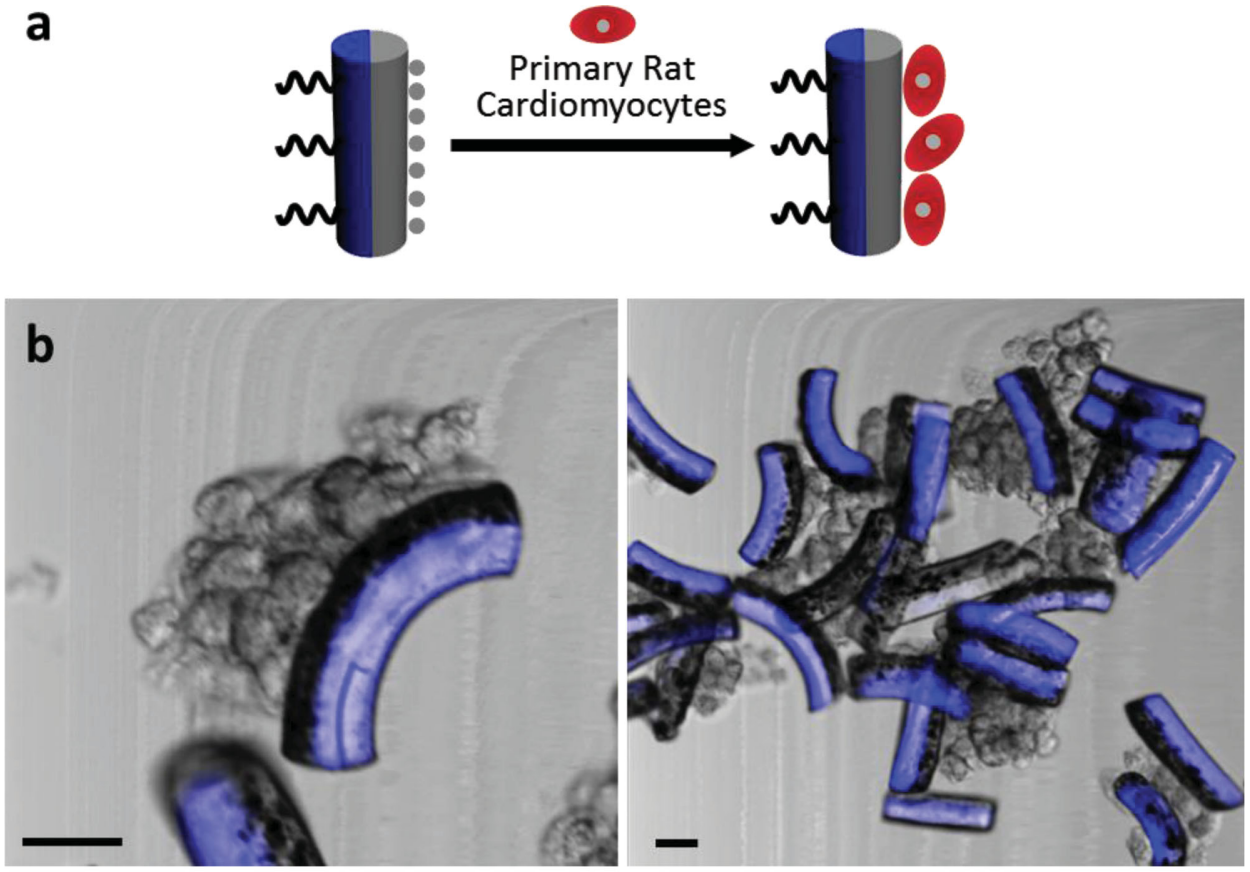

Figure 4. Selective patterning of cardiomyocytes on bicompartmental PLGA/PLCL microcylinders. a) Experimental scheme showing surface modification of one half compartments with PEG and cardiomyocytes seeding of another half compartments. b) The microcylinders showing selective recruitment of cardiomyocytes on the non-PEGylated compartment only. All scale bars are $20 \mu \mathrm{m}$. 
successfully underwent rhythmic bending, which was driven by synchronous beating of the heart cells (Movie S1, Supporting Information). Based on this finding, the actuation of the microcylinders was estimated by analyzing the movies recorded by confocal laser scanning microscopy (CLSM). The bottom end point of the microcylinder was fixed while the displacement of the top end point was measured directly from the video frame (Figure 5).

We found a significant displacement from 9.75 to $18 \mu \mathrm{m}$, confirming a clear bending action of the microcylinder in response to the beating neonatal cardiomyocytes. In fact, the microcylinder's upper displacement was nearly double that of its initial starting point. We note that the stress of this experimental system can be modeled as a micropillar. ${ }^{[45]}$ This approach assumes that the cells bending the microcylinder are selectively exhibiting a net force on the top, which is a reasonable first approximation, and the force needed to bend it from the sides would be even higher according to Tanaka et al. ${ }^{[46]} L$ is the length of the microcylinder, $I$ is the geometric moment of inertia (here $\pi d^{4} / 64$ for a bending bar, where $d$ is the diameter of the microcylinder), $\delta$ is the cylinder's upper displacement, and $E$ is the Young's modulus of the microcylinder. ${ }^{[47]}$ We measured $E$ to be $476 \pm 31 \mathrm{MPa}, L$ is $78.5 \mu \mathrm{m}, d$ is $20 \mu \mathrm{m}, I$ is $7.854 \times 10^{-21} \mathrm{~m}^{4}$, and $\delta$ is $8.25 \mu \mathrm{m}$. Equation 1 is then used to calculate the force of displacement: ${ }^{[45]}$

$F=\left(\frac{3 E I}{L^{3}}\right) \delta$

From this, we get a total force of $191 \pm 12 \mu \mathrm{N}$.

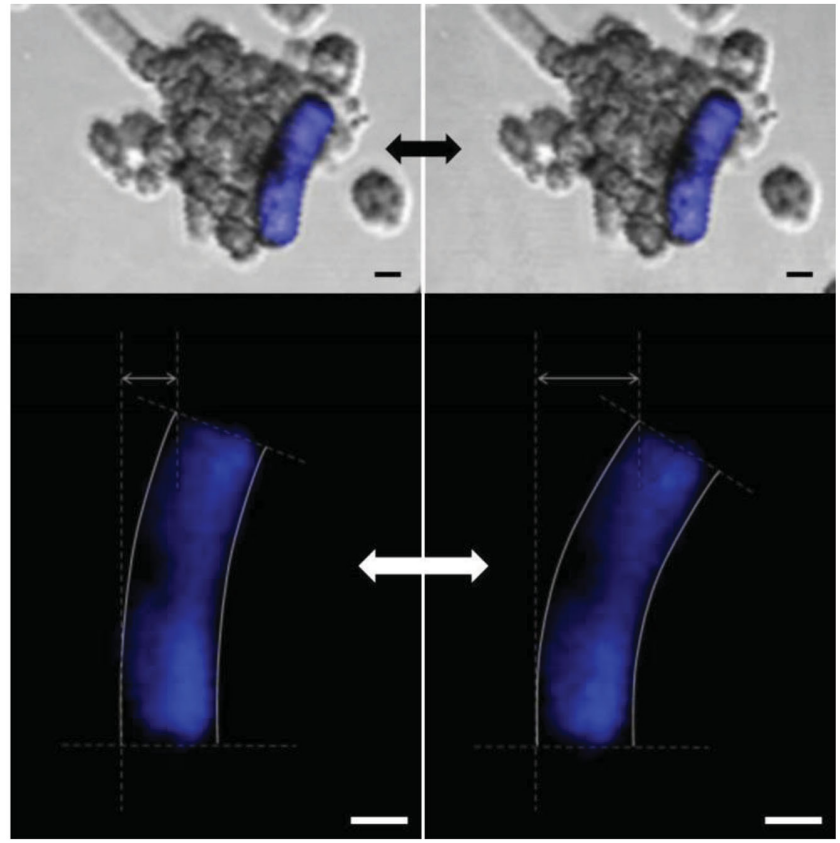

Figure 5. Controlled bending behavior of PLGA/PLCL microcylinders driven by the spontaneous contraction of cardiomyocytes. Two CLSM images were captured from the video frame (top) and analyzed to calculate the displacement of the top end point of the microcylinder (bottom). The displacement was measured from 9.75 to $18 \mu \mathrm{m}$ indicating the deformation of microcylinders was occurred upon the cell movement. All scale bars are $10 \mu \mathrm{m}$.
In summary, a new approach to fabricate biohybrid microcylinders is introduced. From our study, we can conclude that having anisotropic features with nonfouling PEG on a specific compartment significantly influences cardiomyocytes to attach with an aligned direction along the long axis of the microcylinders, which in turn, allows the microcylinders to deform their shapes in a controlled direction. Based on our experiments, we estimated a bending force of $191 \pm 12 \mu \mathrm{N}$. This is a very large force in comparison to those measured for other bioactuators. However, we attribute this exceptional performance that our microactuators have a far greater number of cardiomyocytes per area than other rather macroscopic systems. For example, Akiyama's dorsal vessel tissue robot exerted a force of $4.7 \mu \mathrm{N}$ for bending of their microcylinders (though they were calculating the contracting force at the center of the pillar rather than the top). ${ }^{[47]}$ Tanaka's micropillars were bent with a force of $3.8 \mu \mathrm{N}$, which is unsurprising given that they only have one or a few cardiomyocytes attached. ${ }^{[46]}$ Nishimura et al. averaged the amount of force generated by a single adult rat cardiomyocyte (with a population of 18 measured) with isometric conditions as $5.720 \pm 0.41 \mu \mathrm{N} .{ }^{[48]}$ Visual inspection of the cylinder reveals there are $\approx 26$ cardiomyocytes attached, giving them an average individual force of $\approx 7.3 \mu \mathrm{N}$. This is not too far from the forces measured by Nishimura et al. for a single cardiomyocyte.

While further studies are needed to study the bending behaviors of the heart cells on these structures, the development of a single microcylinder that can be used for the integration of the motion of living cells may open new steps toward miniaturized biorobots or biomachines.

\section{Experimental Section}

Materials: PLGA $\left(85: 15, M_{\mathrm{w}}=50-75000 \mathrm{~g} \mathrm{~mol}^{-1}\right)$, PLCL (lactide $86 \mathrm{~mol} \%)$, Tween 20 , poly[( $m$-phenylenevinylene)-alt-(2,5-dibutoxy- $p$ phenylenevinylene)] (MEHPV), which was used as CLSM markers for blue emission, were purchased from Sigma-Aldrich, USA. COT-PLA was prepared as described elsewhere. Human plasma FN and albumin from bovine serum (BSA) (Alexa Fluor-647 Conjugate) were purchased from Life Technologies, USA. Iron oxide nanocrystals (15 nm in diameter) suspended in chloroform was purchased from Ocean Nanotech, USA, and 8-arm PEG-azide $\left(M_{\mathrm{w}}=40000 \mathrm{~g} \mathrm{~mol}^{-1}\right)$ was purchased from Creative PEGWorks, USA. NIH 3 T3 fibroblasts and primary rat cardiac myocytes were purchased from the American Type Culture Collection (ATCC) and Lonza, USA, respectively. The solvents chloroform, $N, N^{\prime}$. dimethyl formamide (DMF), and cyclohexane were purchased from Sigma-Aldrich, USA and were used without further purification.

Microcylinder Characterization: The particles were visualized using a confocal laser scanning microscopy (Olympus, FluoView 500). A $405 \mathrm{~nm}$ laser and a $633 \mathrm{~nm}$ helium-neon red ( $\mathrm{HeNeR}$ ) laser were used to excite MEHPV and Alexa Fluor 647 (labeling dyes for BSA), respectively. The barrier filters were set to $430-460 \mathrm{~nm}$ for MEHPV and $>660 \mathrm{~nm}$ for Alexa Fluor 647. Confocal Raman spectra were obtained with WITec alpha300R utilizing $532 \mathrm{~nm}$ laser. Microfibers were dried under vacuum for overnight to remove any remaining solvents. The spectra were acquired using an integration time of $0.5 \mathrm{~s}$ per pixel with image scan area of 100 pixels $\times 100$ pixels. The mechanical properties of the microfibers were determined by using Nanolndenter II (MTS/Nanoinstruments Inc., Oak Ridge, TN). Each end point of microfiber was held to a piece of paper with epoxy glue for easy handling and was gripped to a sample holder for stretching. Stress-strain curves were obtained by testing a single fiber with a diameter of $20 \mu \mathrm{m}$ and a length ranges from 2 to 
$5 \mathrm{~mm}$ depending on the sample size. Tensile tests were performed at a rate of $0.08 \mathrm{~mm} \mathrm{~s}^{-1}$. The number of tested samples was 10 for each set.

Fabrication of Bicompartmental Microfibers: The typical procedure for bicompartmental microfibers is as follows. Two different polymer solutions were prepared in separate vials. A $30 \mathrm{w} / \mathrm{v} \%$ of PLGA was dissolved in a solvent mixture of chloroform and DMF $(95: 5, \mathrm{v} / \mathrm{v})$. Another solution contained $30 \mathrm{w} / \mathrm{v} \%$ of PLGA and COT-PLA with a concentration of $9 \mathrm{w} / \mathrm{v} \%$ ( $30 \%$ by weight of PLGA), which were dissolved in the same solvent ratio. More detailed information for the preparation of microfibers created in this work is stated in Table S1 (Supporting Information). The experimental setup includes a syringe pump (Fisher Scientific, Inc., USA), a power supply (DC voltage source, Gamma High Voltage Research, USA), and a rotary collector. Each set of two polymer solutions was delivered at a constant flow rate of $0.05 \mathrm{~mL} \mathrm{~h}^{-1}$ via vertically positioned syringes equipped with $26 \mathrm{G}$ needles (Hamilton Company, USA). When a driving voltage of 11-12 kV was applied to the polymer solution, stable Taylor Cone was formed and fibers were collected at a distance of $7 \mathrm{~cm}$.

Fabrication of Bicompartmental Microcylinders: Bicompartmental microcylinders were prepared as previously reported method. ${ }^{\left[{ }^{[8]} \text { Briefly, }\right.}$ the obtained microfibers (refer to Table S1, Supporting Information) were placed in a cryostat mold with a freezing medium and sectioned at a desired thickness using a cryostat microtome (Microm HM550, Thermo Fisher Scientific Inc., Germany). Once all the sections were collected, the microcylinders were washed extensively with $0.01 \mathrm{v} / \mathrm{v} \%$ Tween 20/deionized (DI) water to remove any residual freezing medium.

Selective Surface Modification via Copper-Free Click Chemistry: COTfunctionalized bicompartmental microfibers were collected on a glass substrate and fixed using double-sided tapes. The glass substrates for holding the microfibers were PEGylated via atom transfer radical polymerization of oligo(ethyleneglyocol) methacrylate. It is important to note that in all cases PEGMA-coated substrate was placed with fiber samples because they were proven to create nonfouling surfaces that can repel both protein adsorption and cell adhesion. ${ }^{[49,50]}$ The microfibers taped on the poly(ethylene glycol) methacrylate (PEGMA)coated substrate were then incubated with $45 \mathrm{mg}$ of 8-arm PEG azide in $1 \mathrm{~mL}$ of DI water and the reaction was carried out in a 12-well plate by gentle agitation for $24 \mathrm{~h}$ at room temperature. After the reaction, the unreacted PEG azide was washed with $0.01 \mathrm{v} / \mathrm{v} \%$ Tween 20/DI water. In the case of microcylinders, $\approx 50000$ cylinders were incubated with $45 \mathrm{mg}$ of 8-arm PEG azide in an Eppendorf tube containing $1 \mathrm{~mL}$ of $0.01 \mathrm{v} / \mathrm{v} \%$ Tween 20/DI water and were kept rotating for $24 \mathrm{~h}$ at room temperature.

BSA Incubation: After the click reaction, the microfibers taped on the PEGMA substrate were placed in a 12-well plate and incubated with $100 \mu \mathrm{g}$ of BSA in phosphate buffered saline (PBS) for $3 \mathrm{~h}$ at room temperature. The unreacted BSA molecules were removed by repeated washing with $0.01 \mathrm{v} / \mathrm{v} \%$ Tween 20/PBS. In the case of microcylinders, 50000 cylinders were rotated with $200 \mu \mathrm{g}$ of BSA in an Eppendorf tube containing $1 \mathrm{~mL}$ of $0.01 \mathrm{v} / \mathrm{v} \%$ Tween 20/PBS for $3 \mathrm{~h}$ at room temperature.

Incubation of NIH3T3 Fibroblasts on Microfibers: Prior to cell seeding, microfibers with PEGMA-coated substrate were first incubated with $50 \mu \mathrm{g}$ of FN in $1 \mathrm{~mL}$ of PBS and gently agitated in a 12-well plate for $1 \mathrm{~h}$ at room temperature. After the FN incubation, the microfibers were washed three times with PBS with an interval of $5 \mathrm{~min}$ in each wash. Next, NIH 3T3 fibroblasts were cultured with a concentration of $5 \times 106$ cells $\mathrm{mL}^{-1}$ well ${ }^{-1}$ for $6 \mathrm{~h}$ in serum-free media. Passages 3-6 were used for all experiments. The cells were then fixed with $4 \%$ paraformaldehyde in DPBS and were stained with $1 \mu \mathrm{L} \mathrm{mL} \mathrm{m}^{-1}$ of cytocalasin D (Life Technologies).

Incubation of NIH3T3 Fibroblasts on Microcylinders: FN (100 $\mu \mathrm{g})$ was incubated with 50000 microcylinders in an Eppendorf tube containing $0.01 \mathrm{v} / \mathrm{v} \%$ PBS at a total volume of $1 \mathrm{~mL}$ and rotated for $1 \mathrm{~h}$ at room temperature. Next, NIH $3 \mathrm{~T} 3$ fibroblasts were cultured with a concentration of $5 \times 106$ cells $\mathrm{mL}^{-1}$ for $4 \mathrm{~h}$ by using a rotator in the incubator. After $4 \mathrm{~h}$ of rotation, the samples were transferred into a 12-well plate with PEGMA surfaces and continued to incubate for another $4 \mathrm{~h}$. Finally, the cells were fixed with $4 \%$ paraformaldehyde in Dulbecco's phosphate buffered saline (DPBS) before imaging.
Incubation of Cardiomyocytes: The PEGMA-coated surfaces and microcylinders were sterilized under UV lamp for overnight at room temperature prior to use. The microcylinders (50 000 cylinders $\mathrm{mL}^{-1}$ ) were first incubated with $\mathrm{FN}$ in $\mathrm{DI}$ water with a concentration of $100 \mu \mathrm{g} \mathrm{mL} \mathrm{m}^{-1}$ in an Eppendorf tube. This was rotated for $1 \mathrm{~h}$ at room temperature. After the FN incubation, the microcylinders were washed three times with sterile DPBS with an interval of $5 \mathrm{~min}$ in each wash. Next, we added primary rat cardiac myocytes (800 000 cells $\mathrm{mL}^{-1}$ ) to the solution containing microcylinders and rotated with media (rat cardiac myocyte basal medium, Lonza) supplemented with 5-bromo$2^{\prime}$-deoxyuridine $(\mathrm{BrdU})$ in order to prevent fibroblast proliferation. After $4 \mathrm{~h}$ of rotation, $80 \%$ of the media was replaced to freshly prepared media with 5-bromo-2'-deoxyuridine (BrdU). The solution was kept rotating for another $4 \mathrm{~h}$ and transferred into a 24-well plate along with the PEGMAcoated surface. After $24 \mathrm{~h}, 1 \mu \mathrm{mol} \mathrm{L} \mathrm{L}^{-1}$ of aldosterone (Sigma-Aldrich) dissolved in ethanol was added to the media in order to enhance cardiomyocyte beating. The samples placed in the well plate were then kept in the incubator until the day 3 and were imaged through CLSM.

\section{Supporting Information}

Supporting Information is available from the Wiley Online Library or from the author.

\section{Acknowledgements}

The authors acknowledge funding from the Multidisciplinary University Research Initiative of the Department of Defense (DOD) and the Army Research Office (W911NF-10-1-0518), the DOD through an idea award (W81XWH-11-1-0111), and the EU-Project SAVVY (310445). The authors acknowledge the Microscopy and Image Analysis Lab (MIL) at the University of Michigan for use of the CLSM and cryostat microtome.

Received: March 17, 2015

Revised: April 27, 2015

Published online: June 24, 2015

[1] P. Zammaretti, M. Jaconi, Curr. Opin. Biotechnol. 2004, 15, 430

[2] Y. Shao, J. Fu, Adv. Mater. 2014, 26, 1494.

[3] R. W. Carlsen, M. Sitti, Small 2014, 10, 3831.

[4] Y. Akiyama, K. Iwabuchi, Y. Furukawa, K. Morishima, Lab Chip 2009, $9,140$.

[5] S. R. Shin, B. Aghaei-Ghareh-Bolagh, X. Gao, M. Nikkhah, S. M. Jung, A. Dolatshahi-Pirouz, S. B. Kim, S. M. Kim, M. R. Dokmeci, X. Tang, A. Khademhosseini, Adv. Funct. Mater. 2014, 24, 6136.

[6] S. H. Ku, M. Lee, C. B. Park, Adv. Healthcare Mater. 2013, 2, 244.

[7] J. Ramón-Azcón, S. Ahadian, M. Estili, X. Liang, S. Ostrovidov, H. Kaji, H. Shiku, M. Ramalingam, K. Nakajima, Y. Sakka, A. Khademhosseini, T. Matsue, Adv. Mater. 2013, 25, 4028.

[8] F. Menaa, A. Abdelghani, B. Menaa, J. Tissue Eng. Regener. Med. 2014, DOI: 10.1002/term.1910.

[9] M. Pilarek, P. Neubauer, U. Marx, Sens. Actuators B 2011, 156, 517.

[10] Z. Boyang, X. Yun, H. Anne, T. Nimalan, R. Milica, Nanotechnology 2011, 22, 494003.

[11] M. M. Stevens, J. H. George, Science 2005, 310, 1135.

[12] R. Ravichandran, S. Sundarrajan, J. R. Venugopal, S. Mukherjee, S. Ramakrishna, Macromol. Biosci. 2012, 12, 286.

[13] A. M. Ross, Z. Jiang, M. Bastmeyer, J. Lahann, Small 2012, 8, 336.

[14] H. Kim, D.-H. Kang, M. Kim, A. Jiao, D.-H. Kim, K.-Y. Suh, Ann. Biomed. Eng. 2012, 40, 1339. 
[15] S. Mandal, S. Bhaskar, J. Lahann, Macromol. Rapid Commun. 2009, 30, 1638.

[16] F. Klein, T. Striebel, J. Fischer, Z. Jiang, C. M. Franz, G. von Freymann, M. Wegener, M. Bastmeyer, Adv. Mater. 2010, 22, 868.

[17] S. M. Berry, S. P. Warren, D. A. Hilgart, A. T. Schworer, S. Pabba, A. S. Gobin, R. W. Cohn, R. S. Keynton, Biomaterials 2011, 32, 1872.

[18] K. Shimizu, H. Araki, K. Sakata, W. Tonomura, M. Hashida, S. Konishi, J. Biosci. Bioeng. 2015, 119, 212.

[19] M. Yoshida, K.-H. Roh, S. Mandal, S. Bhaskar, D. Lim, H. Nandivada, X. Deng, J. Lahann, Adv. Mater. 2009, 21, 4920.

[20] J. Kim, R. C. Hayward, Trends Biotechnol. 2012, 30, 426.

[21] X. Deng, T. W. Eyster, Y. Elkasabi, J. Lahann, Macromol. Rapid Commun. 2012, 33, 640.

[22] J. Y. Lee, S. S. Shah, J. Yan, M. C. Howland, A. N. Parikh, T. Pan, A. Revzin, Langmuir 2009, 25, 3880.

[23] M. S. Hahn, L. J. Taite, J. J. Moon, M. C. Rowland, K. A. Ruffino, J. L. West, Biomaterials 2006, 27, 2519.

[24] K. Y. Suh, J. Seong, A. Khademhosseini, P. E. Laibinis, R. Langer, Biomaterials 2004, 25, 557.

[25] S. Pedron, S. van Lierop, P. Horstman, R. Penterman, D. J. Broer E. Peeters, Adv. Funct. Mater. 2011, 21, 1624.

[26] M. Nikkhah, F. Edalat, S. Manoucheri, A. Khademhosseini, Biomaterials 2012, 33, 5230 .

[27] H. T. H. Au, I. Cheng, M. F. Chowdhury, M. Radisic, Biomaterials 2007, 28, 4277

[28] N. Bursac, K. K. Parker, S. Iravanian, L. Tung, Circ. Res. 2002, 91 e45.

[29] Y. Tanaka, K. Morishima, T. Shimizu, A. Kikuchi, M. Yamato, T. Okano, T. Kitamori, Lab Chip 2006, 6, 230.

[30] J. Park, I. C. Kim, J. Baek, M. Cha, J. Kim, S. Park, J. Lee, B. Kim, Lab Chip 2007, 7, 1367.

[31] J. Kim, J. Park, S. Yang, J. Baek, B. Kim, S. H. Lee, E.-S. Yoon, K. Chun, S. Park, Lab Chip 2007, 7, 1504.

[32] V. Chan, J. H. Jeong, P. Bajaj, M. Collens, T. Saif, H. Kong, R. Bashir, Lab Chip 2012, 12, 88.

[33] S. V. Anand, M. Yakut Ali, M. T. A. Saif, Lab Chip 2015, 15, 1879.
[34] A. W. Feinberg, A. Feigel, S. S. Shevkoplyas, S. Sheehy, G. M. Whitesides, K. K. Parker, Science 2007, 317, 1366.

[35] J. C. Nawroth, H. Lee, A. W. Feinberg, C. M. Ripplinger M. L. McCain, A. Grosberg, J. O. Dabiri, K. K. Parker, Nat. Biotechnol. 2012, 30, 792.

[36] N. Annabi, S. Selimovic, J. P. Acevedo Cox, J. Ribas, M. Afshar Bakooshli, D. Heintze, A. S. Weiss, D. Cropek, A. Khademhosseini, Lab Chip 2013, 13, 3569.

[37] C. Cvetkovic, R. Raman, V. Chan, B. J. Williams, M. Tolish, P. Bajaj, M. S. Sakar, H. H. Asada, M. T. Saif, R. Bashir, Proc. Natl. Acad. Sci. USA 2014, 111, 10125

[38] S. Bhaskar, J. Hitt, S.-W. L. Chang, J. Lahann, Angew. Chem. Int. Ed. 2009, 48, 4589.

[39] S. Rahmani, S. Saha, H. Durmaz, A. Donini, A. C. Misra, J. Yoon I. Lahann, Angew. Chem. Int. Ed. 2014, 53, 2332.

[40] B. L. Seal, T. C. Otero, A. Panitch, Mater. Sci. Eng. R 2001, 34, 147

[41] M. C. Serrano, E. J. Chung, G. A. Ameer, Adv. Funct. Mater. 2010 20, 192.

[42] S. I. Jeong, B.-S. Kim, S. W. Kang, J. H. Kwon, Y. M. Lee, S. H. Kim Y. H. Kim, Biomaterials 2004, 25, 5939.

[43] L. Wang, Z. Zhang, H. Chen, S. Zhang, C. Xiong, J. Polym. Res. 2010, 17, 77 .

[44] Q. Chen, S. Liang, G. A. Thouas, Prog. Polym. Sci. 2013, 38, 584.

[45] J. L. Tan, J. Tien, D. M. Pirone, D. S. Gray, K. Bhadriraju, C. S. Chen, Proc. Natl. Acad. Sci. USA 2003, 100, 1484.

[46] Y. Tanaka, K. Morishima, T. Shimizu, A. Kikuchi, M. Yamato, T. Okano, T. Kitamori, Lab chip 2006, 6, 230.

[47] Y. Akiyama, K. Iwabuchi, Y. Furukawa, K. Morishima, Lab Chip 2009, $9,140$.

[48] S. Nishimura, S. Yasuda, M. Katoh, K. P. Yamada, H. Yamashita Y. Saeki, K. Sunagawa, R. Nagai, T. Hisada, S. Sugiura, Am. J. Physiol. Heart Circ. Physiol. 2004, 287, H196.

[49] X. Jiang, H. Y. Chen, G. Galvan, M. Yoshida, J. Lahann, Adv. Funct. Mater. 2008, 18, 27.

[50] T. D. Y. Kozai, N. B. Langhals, P. R. Patel, X. Deng, H. Zhang, K. L. Smith, J. Lahann, N. A. Kotov, D. R. Kipke, Nat. Mater. 2012, 11, 1065. 